\title{
Study of PSO-RBF Neural Network in Power System Load Prediction
}

\author{
Ai-hua Jiang ${ }^{1, a}$, Yan $\mathrm{Li}^{2}$ and Chen Xue ${ }^{3}$ \\ ${ }^{1}$ Guangxi key Laboratory of Power System Optimization and Energy Technology, Guangxi \\ University, Nanning, 530004, China; \\ ${ }^{2}$ Yulin Power Supply Bureau, Guangxi Power Grid, Yulin, 537000, China; \\ ${ }^{3}$ Maintenance Company, State Grid Sichuan Electric Power Corporation, Chengdu, 610000, China. \\ ajiangaihua2229@126.com
}

Keywords: RBF, PSO, Power system, Load prediction.

\begin{abstract}
Short-term load prediction of power system has great significance for safety and economy of power system operation as basic content of power system operation management and real-time control. In the paper, power system short-term load predicting model based on RBF neural network was established. Influences of temperature, holidays and other factors on power system load were mainly considered in the model. PSO optimization algorithm was adopted for optimizing initial weights and base width of RBF neural network aiming at random settings of initial weights and base width of RBF neural network. History real load data was verified, and the verified results were compared with traditional RBF neural network model, the results showed that the prediction precision of RBF neural network model optimized by PSO algorithm was obviously improved, thereby providing an effective method for short-term load prediction of power system.
\end{abstract}

\section{Introduction}

Short-term load prediction of power system has great significance for safety and economy of power system operation as basic content of power system operation management and real-time control with constant improvement of power system automation. However, since the power system load is characterized by strong randomness of change trend, various influence factors, etc., it is difficult to establish effective and uniform predication model for power system load prediction [1]. Traditional load predicting techniques have been unable to meet higher and higher requirements on load prediction precision of power sector. Therefore, it has great significance to search new load prediction method on economic operation and automatic scheduling of power system [2]. Neural network is a subject which was developed rapidly at the end of the twentieth century. Since neural network has good nonlinear mapping ability, self-learning adapting ability and parallel information processing ability, it provides new thought and methods for identifying unknown and uncertain systems [3]. Radial Basis Function (RBF) neural network is a kind of feed-forward neural network with single hidden layer, which belongs to a neural network of three-layer structure for simulating human brain for locally adjusting mutually covered receptive fields. It was proved that RBF neural network can approximate any contact function with arbitrary precision. Therefore, it is widely applied in power system load prediction. However, RBF neural network is neutral network of local approximation. Therefore, it is critical to determine initial weights. Random generation mode is adopted for determining initial weights and base width of traditional RBF neural network, thereby affecting identification precision of RBF neural network [4]. In the paper, Particle Swarm Optimization algorithm (PSO) can be adopted for optimally selecting initial weights and base width of RBF neural network, thereby realizing global search of RBF neural network and improving prediction precision of RBF neural network for power system load. 


\section{Establishment of RBF Neural Network Prediction Model}

Power Load Modeling. Power system load prediction belongs to time series prediction. There are two methods for predicting time series generally: one is parameter prediction method, and the other is non-parameter prediction method. Time series prediction by neural network belongs to non-parameter prediction method, it does not need accurate system model, therefore it has wider application scope compared with parameter prediction methods. Time series prediction can be regarded as mathematics mapping for calculating future trend with historical record. Mathematical model for predicting future trend by $\mathrm{k}$ steps in advance through $\mathrm{m}$ point historical data is shown in formula (1).

$$
X(i), X(i+1), \cdots, X(i+k)=p(X(i-1), X(i-2), X(i-m))
$$

Wherein: $i=m+1, m+2, \cdots,(N-m-k) / s$ is size of study sample; $\mathrm{N}$ is the length of sample data; $\mathrm{s}$ refers to sliding point quantity (it is 1 generally). Because $\mathrm{L}-\mathrm{M}$ neutral network can approximate the mapping relationship implied in the sample data, L - M neural network optimized by genetic algorithm was adopted for solving the problem of time series prediction.

Identification of RBF Neural Network on Power Load Model. RBF neural network belonged to a three-layer forward network, mapping from input to output was nonlinear, and mapping from hidden layer space to output space was linear. In addition, RBF belonged to neural network of local approximation. Therefore, identification of RBF neural network on RBF neural network can greatly accelerate network learning speed, and local minimum problem can be locally avoided [5-6]. The structure of RBF neural network was shown in figure 1.

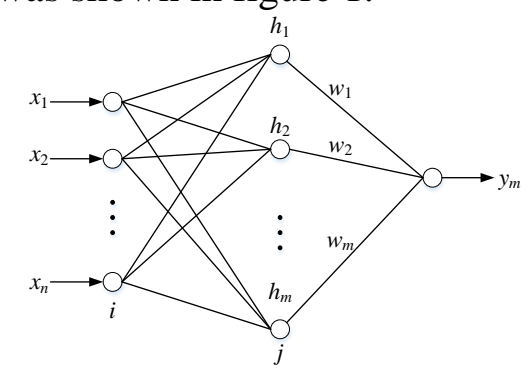

Fig. 1 RBF Neural Network Structure

Structure diagram of identifying power system model with RBF neural network was shown in figure 2. $u(k)$ belonged to input signal, $y(k)$ referred to output signal, $y_{m}(k)$ indicated output of RBF neural network, error signal between output of RBF neural network and real output of power system model was regarded as input signal of learning algorithm for self-learning of RBF neural network, thereby realizing learning and identification of RBF neural network on power system model.

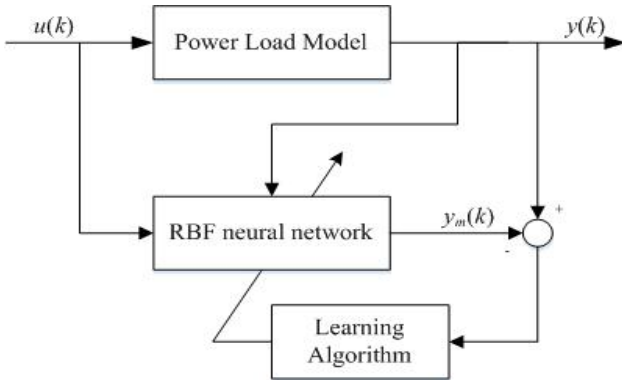

Fig. 2 RBF Neural Network Approximation

In RBF neural network structure, $X=\left[x_{1}, x_{2}, \cdots x_{n}\right]^{T}$ belonged to input quantity of network. $H=\left[h_{1}, h_{2}, \cdots h_{m}\right]^{T}$ referred to radial basis vector, wherein $h_{j}$ was Gaussian basis function, and $h_{j}$ calculation was shown as formula (2).

$$
h_{j}=\exp \left(-\frac{\left\|X-C_{j}\right\|^{2}}{2 b_{j}^{2}}\right), j=1,2, \cdots, m
$$

In the formula, $C_{j}$ represented center vector of the $j$ th node in the hidden layer, in addition, $C_{j}=\left[c_{j 1}, c_{j 2}, \cdots, c_{j n}\right]^{T}, j=1,2, \cdots, n$. 
Base width vector of RBF neural network was $B=\left[b_{1}, b_{2}, \cdots b_{m}\right]^{T}, b_{j}$ referred to base model parameters of node $j$. The weight from input layer to hidden layer in RBF neural network was 1 . Weight from hidden layer to output layer was $W=\left[w_{1}, w_{2}, \cdots w_{m}\right]^{T}$. Output of RBF neural network can be calculated as shown in formula (3).

$$
y_{m}(k)=w_{1} h_{1}+w_{2} h_{2}+\cdots+w_{m} h_{m}
$$

Error index function of RBF neural network identification was expressed as $E(k)=1 / 2\left(y(k)-y_{m}(k)\right)^{2}$, thus RBF neural network output power can be obtained. Updating formula of node base model parameters and node center vector is shown in formula (4) - (8).

$$
\begin{aligned}
w_{j}(k)=w_{j}(k-1)+\eta\left(y(k)-y_{m}(k)\right) h_{j}+\alpha\left(w_{j}(k-1)-w_{j}(k-2)\right) \\
\Delta b_{j}=\left(y(k)-y_{m}(k)\right) w_{j} h_{j} \frac{\left\|X-C_{j}\right\|^{2}}{2 b_{j}^{2}} \\
b_{j}(k)=b_{j}(k-1)+\eta \Delta b_{j}+\alpha\left(b_{j}(k-1)-b_{j}(k-2)\right) \\
\Delta c_{j i}=\left(y(k)-y_{m}(k)\right) w_{j} h_{j} \frac{x_{i}-c_{j i}}{2 b_{j}^{2}} \\
c_{j i}(k)=c_{j i}(k-1)+\eta \Delta c_{j i}+\alpha\left(c_{j i}(k-1)-c_{j i}(k-2)\right)
\end{aligned}
$$

In the formula, $\eta$ referred to learning efficiency, $\alpha$ referred to momentum factor, and values of $\eta$ and $\alpha$ were between 0 and 1 .

\section{PSO Algorithm Principle}

PSO algorithm refers to a global random search optimization algorithm based on swarm intelligence, which was proposed by Kennedy and Eberhart and inspired by flock migration and clustering behavior in foraging process. In PSO algorithm, a group of initial solutions were randomly initialized firstly, the optimal solution of problems can be finally obtained through iterative optimization.. In each iteration process, each particle should maintain two values to update itself. One was the optimal solution searched by particles themselves, the other was the optimal solution searched by the entire population.

We assumed that one species was composed of $N$ particles in one D-dimensional goal search space in order to derive optimization process of particle swarm, wherein the $i$ th particle can be expressed with one D-dimensional vector.

$$
X_{i}=\left(x_{i 1}, x_{i 2}, \cdots, x_{i D}\right), \quad i=1,2, \cdots, N
$$

Movement speed of the $i$ th particle was also a D-dimensional vector, which was recorded as follows:

$$
V_{\mathrm{i}}=\left(v_{i 1}, v_{i 2}, \cdots, v_{i D}\right), \quad i=1,2, \cdots 3
$$

The optimal position currently searched by the $i$ th particle was defined as individual extremum, which was recorded as follows:

$$
p_{\text {best }}=\left(p_{i 1}, p_{i 2}, \cdots, p_{i D}\right), \quad i=1,2, \cdots, N
$$

The optimal position currently searched by the group was defined as global extremum, which was recorded as follows:

$$
g_{\text {best }}=\left(p_{g 1}, p_{g 2}, \cdots, p_{g D}\right)
$$

Each particle in the population can update own speed and position according to the following formula (13) and (14) after the two optimal values were obtained:

$$
\begin{gathered}
v_{i d}=w * v_{i d}+c_{1} r_{1}\left(p_{i d}-x_{i d}\right)+c_{2} r_{2}\left(p_{g d}-x_{i d}\right) \\
x_{i d}=x_{i d}+v_{i d}
\end{gathered}
$$

In formula (13) and formula (14), $c_{1}$ and $c_{1}$ were acceleration constants, $r_{1}$ and $r_{1}$ were uniform random numbers within scope $[0,1] . c_{1}=c_{2}=2 i=1,2, \cdots, D$ were generally selected according to 
long-term experience accumulated by human beings. $v_{i d}$ referred to updating speed of particles. $v_{i d} \in\left[-v_{\max }, v_{\max }\right], v_{\max }$ was constant, which was used for restricting updating speed of particles. Flow chart of particle swarm optimization algorithm can be summarized as the following six steps. Specific optimization process was shown in figure 3.

(1) Particle swarm was initialized, including population size $N$, position $x_{i}$ and velocity $V_{\mathrm{i}}$ of each particle;

(2) Fitness value $F_{i t}[i]$ of each particle was calculated;

(3) Individual extremum was updated, each particle in the population compared own fitness value $F_{i t}[i]$ with individual extremum $p_{\text {best }}(i)$. If $F_{i t}[i]>p_{\text {best }}(i)$, own fitness value Fit $[i]$ was used to replace current individual extremum $p_{\text {best }}(i)$;

(4) Global extremum was updated, each particle in the population compared with fitness value Fit $[i]$ with global extremum $g_{\text {best }}$. If $F_{i t}[i]>p_{\text {best }}(i)$, own fitness value $F_{i t}[i]$ was used to replace current global extremum $g_{\text {best }}$;

(5) Current speed $v_{i}$ and current position $v_{i}$ of particle were updated according to formula (12) and formula (13).

(6) The system retreated if ending conditions were met (error met the requirement or reached the maximum iteration frequency), otherwise the system returned to (2) for continuous implementation.

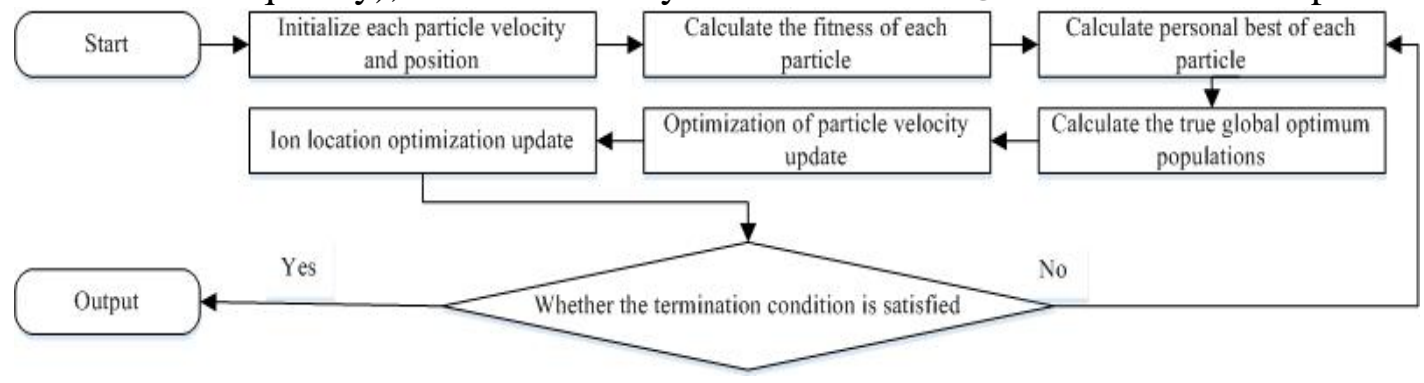

Fig.3 Flow Chart of PSO Algorithm

\section{Simulation of PSO-RBF Neural Network in Power System Load Prediction Algorithm}

In the paper, PSO optimization algorithm was adopted for optimizing initial weights and base width of RBF neural network in order to overcome defects of RBF neural network in power system model identification process, such as local optimum and initial weights as well as base width random initialization. Global feature of PSO optimization algorithm was utilized for remedying local identification features of RBF neural network. Meanwhile, the optimal base width and initial weights were given, and identification precision of RBF neural network on power system model was effectively improved, thereby providing model basis for predicating power system load.

Settings of PSO - RBF Neural Network Prediction. Kolmogorov theorem showed that three-layer forward neural network can approximate any continuous nonlinear function. Therefore, three-layer structure was adopted for neutral network of PSO-RBF neural network load prediction model in the paper, namely one input layer, one hidden layer and one output layer. The input layer contained 26 neurons, 1-14 input indicated previous load values of power system in the former day, and the 25th input represented temperature factor, and the $25^{\text {th }}$ input represented holiday factor. Hidden layer contained 5 neurons. Output layer contained 24 neurons, which represented predicted value of power lad. The learning rate parameter was 0.85 , and the momentum factor was 0.05 . In PSO optimization algorithm, the dimension of the particles was 153, wherein 1-3 dimension represented base width parameter, 4-81 dimension represented center vector of node, and 82-153 dimension represented weights of output layer. PSO specific parameter settings were shown in table 1.

Load Prediction Simulation of PSO-RBF Neural Network Power System. In the paper, 100 groups of real data in some transformer substation was utilized for training and load prediction on RBF neural network model. Before power system model was identified by RBF neural network, 
initial weights and base weight of RBF neutral network were optimized through concrete strategies of PSO optimization RBF neural network proposed in the paper, and the error convergence curve of PSO optimization algorithm is shown in Figure 4.

Table 1 Parameter Setting of PSO Optimization RBF Neural Network

\begin{tabular}{ll}
\hline PSO optimization algorithm parameter & Value \\
\hline Population particle number & 20 \\
Particle dimension & 153 \\
Iteration frequency & 250 \\
Inertia weight & 0.1 \\
Parameter $\mathrm{c}_{1}$ & 2 \\
Parameter $_{2}$ & 2 \\
\hline
\end{tabular}

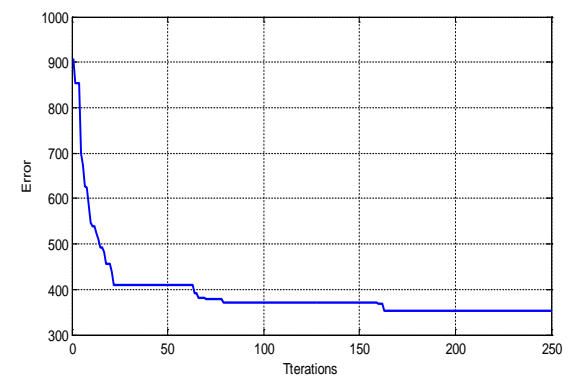

Fig. 4 Error Convergence Curve of PSO to Optimize RBF Neutral Network

Error Convergence Curve of PSO to Optimize RBF Neutral Network showed that the error of optimized initial weights and base width was greatly reduced compared with error of random values. After initial weights and base width of RBF neural network were optimized, RBF neutral network can be utilized for predicating power system load, and the predication results shown shown in figure 5 .

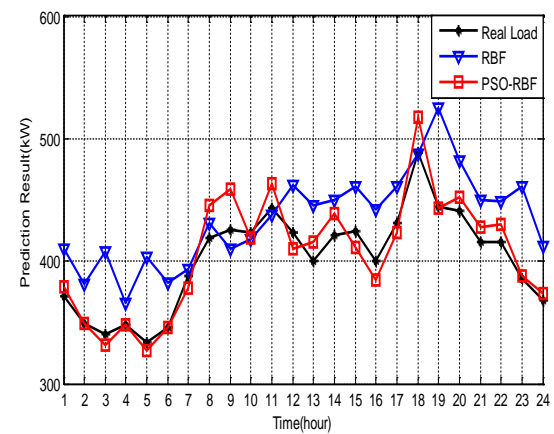

Fig.5 Prediction Effect of Power System Load

In the paper, RBF neural network based on PSO algorithm was compared with prediction effect of traditional RBF neural network on power system load in order to verify the prediction precision of RBF neural network based on PSO algorithm in the paper. Prediction error of both RBF neural networks is shown in figure 6.

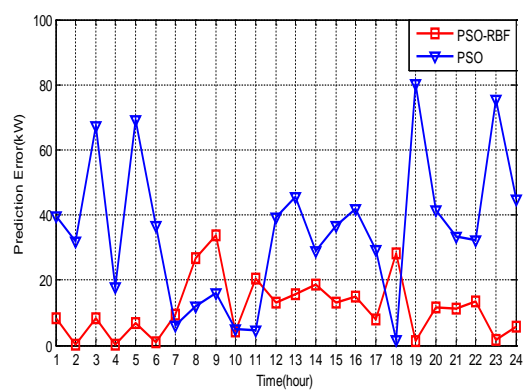

Fig. 6 Prediction Error of Power System Load

Prediction error stimulation results of RBF neural network on power system load showed that prediction precision of RBF neural network based on PSO algorithm for power system load was much better than traditional RBF neural network in the paper. Since traditional RBF neural network 
can randomly set network initial weights and base width, identification error of traditional RBF neural network on power load model was larger, and then prediction precision of power system and load was affected.

In the paper, prediction error of two RBF neural networks for power system load was counted in order to more intuitively compare prediction precision of RBF neural network based on PSO algorithm in the paper with the prediction precision of RBF neural network. and the statistical results were shown in table 2.

Table 2 Prediction Error of RBF Neural Network on Power System Load

\begin{tabular}{ll}
\hline RBF Neutral Network & Accumulated Error \\
\hline Traditional RBF Neutral Network & 846.6 \\
PSO-RBF Neutral Network & 201.9 \\
\hline
\end{tabular}

Prediction error of RBF neural network on power system load in Table 2 showed that prediction error of RBF neural network based on PSO algorithm in the paper was 201.9, which was prominently better than the prediction error of traditional RBF neutral network 846.6. The prediction effect was prominently improved. The above stimulation results showed that initial weights and base width optimization strategy of RBF neutral network based on PSO algorithm in the paper can effectively improve identification precision of RBF neural network on power load model, thereby improving prediction precision on power system load. It has wide realistic significance on dispatching power load.

\section{Conclusion}

In the paper, power system short-term load prediction model based on RBF neural network was established. Influence of temperature, holidays and other factors on power system load were mainly considered in the model. PSO optimization algorithm was adopted for optimizing initial weights and base width of RBF neural network aiming at random settings of initial weights and base width of RBF neural network. History real load data was verified, and the verified results were compared with traditional RBF neural network model, the results showed that the prediction precision of RBF neural network model optimized by PSO algorithm was obviously improved, thereby providing an effective method for short-term load prediction of power system.

\section{References}

[1] Li Yanfang. Power load prediction. Journal of Nanchang College, 2008, 22 (6): 143-145.

[2] Chen Yaowu, Wang Leyu. Short-term power load prediction model based on combined neural network. Proceedings of CSEE, 2001, 21 (4): 79-82.

[3] Zhang Tao, Zhao Dengfu. Short-term load prediction method based on RBF neural network and expert system. Journal of Xi 'an Jiaotong University, 2001, 34 (4): 331-334.

[4] Jiang Aihua, Nixiao Liang. Short-term load forecasting using support vector machine optimized by the improved fruit fly algorithm and the similar day method. Electricity Distribution (CICED), 2014 China International Conference on. IEEE, 2014.

[5] Lei Shaolan, Sun Caixin, Zhou Quan, etc. Power short-term load prediction method based on radial neural network and adaptive neural fuzzy system. Proceedings of CSEE, 2005, 22.

[6] Li Lina, Gan Xiaoye, Xu Panfeng, etc. Nonlinear system modeling of improved particle swarm optimization Takagi Sugeno fuzzy radial basis function neural network. Computer Application, 2014, 34 (5): 1341-1344. 